\title{
Mechanical model of sphincterotomy: a step forward for trainees
}

\section{다)(요 $\odot$}

\author{
Authors \\ Jérémie Jacques ${ }^{1,2}$, Romain Legros ${ }^{1}$, Denis Sautereau ${ }^{1}$ \\ Institutions \\ 1 Gastroenterology and Endoscopy Unit, Dupuytren \\ University Hospital, Limoges, France \\ 2 Bio-Em, Xlim, Limoges, France \\ Bibliography \\ DOI https://doi.org/10.1055/a-0830-4712 | \\ Endoscopy International Open 2019; 07: E762-E763
}

\author{
(c) Georg Thieme Verlag KG Stuttgart · New York \\ eISSN 2196-9736 \\ Corresponding author \\ Jérémie Jacques, MD, service d'Hépato-gastro-entérologie, \\ CHU Dupuytren 87042, Limoges, France \\ Fax: +33555058733 \\ jeremiejacques@gmail.com
}

Proficiency in a highly skilled procedure can be a challenge. The classical mentor-based approach has increasingly been shown to have its limitations, particularly from an ethical point of view. "Never for the first time in a human" is an important adage that is now possible with the availability of excellent simulation training models.

In this issue of EIO, Prof. Bruno and his team evaluate a new synthetic papilla product that is easily insertable into the Boškoski-Costamagna endoscopic retrograde cholangiopancreatography (ERCP) mechanical simulator [1]. These mechanical simulators have recently been introduced in ERCP training and enable trainees to practice cannulation, stent placement in both the common bile duct (CBD) and pancreatic duct (PD) as well as CBD stone extraction [2,3]. These simulators also allow for demonstration of specific endoscopic movements used in ERCP for the straight position or long route position. Furthermore, the new synthetic papilla feature allows trainees to perform a simulated sphincterotomy. Forty international specialist physicians who have collectively performed more than 2500 ERCPs unanimously concluded that the synthetic papilla feature was a useful tool for trainee endoscopists and should be incorporated into training curricula.

It is a well-established pedagogical theory that there are three characteristic phases to learning a skill: cognitive, associative and, finally, autonomous. Training in and repetition of a procedure are essential in for progression from the cognitive phase, where the cognitive load is intense, to the autonomous phase where the procedure can be repeated by muscle memory. Regular feedback with a mentor needs to be strongly associated with ERCP training programs to optimize the efficiency and success rates of the training process. Training models provide an ideal, realistic environment to allow for this necessary repetition [4] to reach learning outcomes that are procedural, but also trainee-dependent.

Since the $1990 \mathrm{~s}$, a large number of publications have reported positive outcomes with training models that encourage use of endoscopy in both basic and highly skilled procedures. The ex vivo EASIE model has been the most studied, showing improved skills in hemostasis [5], endoscopic ultrasound-guided fine-need aspiration [6], EUS pancreatic collection drainage [7], and ESD. However, this model is associated with significant costs and requires a dedicated structure and animal-dedicated scopes. An in vivo animal model using pigs is one of the best tools for many highly skilled endoscopic procedures, particularly because of the presence of peristalsis and respiratory movements [8]. Availability is, of course, limited by cost, the requirement for a veterinary lab, and compelling ethical considerations. Virtual reality simulation is being used more and more, with 18 published trials to date [9], confirming its suitability for beginners, in particular, to acquire basic gastroscopy and colonoscopy skills. Computer-based simulator systems are expensive but do not need the dedicated structures required in ex vivo or in vivo models. Mechanical simulators provide promising new opportunities. The Thompson Endoscopic Skills Trainer (TEST) allows teaching of basic skills (retroflexion, knob control, torque, polypectomy and navigation/loop reduction) and has a validated metric evaluation system to monitor trainees and observe their progression [10].

However, due to the growing of various training models and potential business linked, learned societies have to think of official validation. Training on a model is attractive but probably not training on any model!

As with skills associated with sports or music, repetition and training are essential to learn and master a series of movements. Given growing evidence of the benefits of this ap- 
proach, simulation platforms dedicated to gastrointestinal endoscopy should be available in teaching hospitals. Metric-validated evaluation tools should also be further developed to regularly evaluate and validate, the procedural ability of trainees. A procedural ability curriculum should not simply be based on a minimum number of procedures that have to be successfully performed under supervision. Models could help not only with regard to skills training but also in evaluation of trainees, particularly on highly skilled procedures (e.g., ERCP, EUS-guided biliary drainage, endoscopic mucosal resection, endoscopic submucosal dissection, and submucosal endoscopy). More specialized physicians, who are akin to high-level sportsmen who regularly practice their skills, could certainly benefit from these models. From this, a procedural Continuous Medical Education system could be imagined.

"Never the first time in a human" and "Never stop training" are two adages that need to be routinely incorporated into the philosophy of a gastrointestinal endoscopy unit for the benefit of their patients. Financial support is needed to shift training from a master-apprentice model to a simulator-based apprentice model on models that have previously been officially validated.

\section{Competing interests}

None

\section{References}

[1] van der Wiel SE, Koch AD, Bruno MJ. Face validity of a synthetic papilla designed for biliary sphincterotomy training. Endosc Int Open 2019; 7: E757-E761

[2] Boškoski I, Costamagna G. The Boškoski-Costamagna ERCP Trainer: from dream to reality. Endoscopy 2016; 48: 593

[3] van der Wiel SE, Koch AD, Bruno M]. Face and construct validity of a novel mechanical ERCP simulator. Endosc Int Open 2018; 6: E758 E765

[4] van der Wiel SE, Küttner Magalhães R, Rocha Gonçalves CR et al. Simulator training in gastrointestinal endoscopy - From basic training to advanced endoscopic procedures. Best Pract Res Clin Gastroenterol 2016; 30: $375-387$

[5] Hochberger ], Matthes K, Maiss J et al. Training with the compactEASIE biologic endoscopy simulator significantly improves hemostatic technical skill of gastroenterology fellows: a randomized controlled comparison with clinical endoscopy training alone. Gastrointest Endosc 2005; 61: 204-215

[6] Gonzalez JM, Cohen J, Gromski MA et al. Learning curve for endoscopic ultrasound-guided fine-needle aspiration (EUS-FNA) of pancreatic lesions in a novel ex-vivo simulation model. Endosc Int Open 2016; 4: E1286-E1291

[7] Moryoussef F, Leblanc S, Bertucat A et al. Comparative evaluation of two porcine ex vivo models for training in endoscopic ultrasoundguided drainage of pancreatic fluid collections. Endosc Int Open 2017; 5: E1020-E1026

[8] Jacques ], Legros R, Charissoux A et al. A local structured training program with live pigs allows performing ESD along the gastrointestinal tract with results close to those of Japanese experts. Dig Liver Dis 2016; 48: $1457-1462$

[9] Khan R, Plahouras J, Johnston BC et al. Virtual reality simulation training for health professions trainees in gastrointestinal endoscopy. Cochrane Database Syst Rev 2018; 8: CD008237

[10] Jirapinyo P, Kumar N, Thompson CC. Validation of an endoscopic parttask training box as a skill assessment tool. Gastrointest Endosc 2015; 81: $967-973$ 